Project Number: 55118 TITLE: Mechanisms of Heavy Metal Sequestration in Soils: Plant-Microbe Interactions and Organic Matter Aging

Annual Report June 25, 2001

Lead Investigator: Teresa W.-M. Fan

530-752-1450

Dept. of Land, Air \& Water Resources, Univ. of California, Davis

twfan@ucdavis.edu

Co-Investigators: $\quad$ Richard M. Higashi

530-752-1450

Crocker Nuclear Laboratory, Univ. of California, Davis

rmhigashi@ucdavis.edu

David Crowley

909-787-3785

Dept. Environmental Sciences, University of California, Riverside crowley@mail.ucr.edu

Research Staff: $\quad$ Terry James

Graduate Student: $\quad$ Anyou Wang

Research Objective

The myriad of human activities including strategic and energy development at various DOE installations have resulted in the contamination of soils and waterways that can seriously threaten human and ecosystem health. Development of efficacious and economical remediation technologies is needed to ameliorate these immensely costly problems. Bioremediation (both plant and microbe-based) has promising potential to meet this demand but still requires advances in fundamental knowledge. For bioremediation of heavy metals, the three-way interaction of plant root, microbial community, and soil organic matter (SOM) ${ }^{1}$ in the rhizosphere is critically important for long-term sustainability but often underconsidered. Particularly urgent is the need to understand processes that lead to metal ion stabilization in soils, which is crucial to all of the goals of bioremediation: removal, stabilization, and transformation. This project will build on the knowledge that we have generated on the role of root exudation and metabolism for metal mobilization and accumulation, to address the following objectives:

1) Identify molecular markers and characterize the chemical nature of recalcitrant SOM pools that are involved in belowground metal ion interactions, which are likely to be markers for sustainable sequestration;

2) Utilize 1) to determine plant and microbial factors that contribute to sustainable metal sequestration or mobility, as well as bioavailability;

3) Utilize information from 1) and 2) to explore efficacious means for enhancing sustainable phytostabilization of heavy metals in the subsurface zone.

Research Progress and Implications 
We have designed a soil aging experimental system capable of stable operation for several months, and used it to run an experiment to investigate the effect of organic amendments on soil organic matter transformation and heavy metal leaching. An agricultural soil (Ag) low in $\mathrm{Cd}$ and $\mathrm{Pb}$ concentrations and a $\mathrm{Cd}-\mathrm{Pb}$ contaminated soil from McClellan Air Force Base (AFB) were amended with wheat straw and cow manure. These soils were allowed to age for 25 weeks during which microbial activities (as $\mathrm{CO}_{2}$ and $\mathrm{N}$ emissions), and metal ion leaching were monitored. Chemical profiles of aging soil organic matter were also acquired using pyrolysis GC-MS after 9 and 25 weeks. Initial analysis indicated that $\mathrm{NH}_{3}$ emission was higher in manure-amended soils (both $\mathrm{Ag}$ and $\mathrm{AFB}$ ) than the wheat straw-amended counterparts while the opposite was observed for the total amounts of organic carbon leached. The enhanced $\mathrm{C}$ leaching with wheat straw treatment may be correlated with the somewhat higher $\mathrm{Cd}$ leaching from this treatment.

With regards to organic matter profiles, the pyro-GC-MS revealed a cacophony of detailed changes, as expected. Examining directly the markers for peptide bonds (reconstructed ion chromatograms, or RIC, for masses 79, 93, and $103 \mathrm{Da}$ ), all soils showed increases, which were less for the AFB than the $\mathrm{Ag}$ soil experiments. One interpretation of the increases of peptides is that it is due to increases in microbial biomass; however, at this juncture, it is not possible to assign the relative peptide amounts in live microbes vs. organic matter. In contrast, lignin residues (revealed thru the RIC of 124, 135, and $151 \mathrm{Da}$; see Figure 1) is interpretable as organic matter, since microbes do not produce lignin structures. Both wheat straw and manure amendments added intense lignin markers to the soils. As illustrated in Figure 1 for a pair of experiments, the lignin marker intensity declined over $90 \%$ for the $\mathrm{Ag}$ soil, but not for the AFB soil. One possible interpretation of this result is that the Cd-contaminated AFB soil failed to promote the lignin-degraders, despite the fact that both soils received the same major organic carbon source. Thus, this approach is poised to provide comparative, biogeochemical information with regards to the microbial community analyses described below.

A separate aging experiment has also been ongoing for 34 weeks, where ${ }^{13} \mathrm{C}$-glucose and ${ }^{15} \mathrm{~N}$-nitrate were incubated with soils from Savannah River Site (SRS) to genearate ${ }^{13} \mathrm{C}$ and ${ }^{15} \mathrm{~N}$-lableled humic substances. (HS). Pyrolysis GC-MS analysis of the soils indicates that various substructures of HS (polysaccarides, peptides, lignin) have been strongly labeled (e.g., >90\% isotopic substitution in peptide backbone carbons). These labeled soils will be useful for following the turnover rate of HS structures, thereby allowing their recalcitrant properties to be characterized as a function of chemical class. 


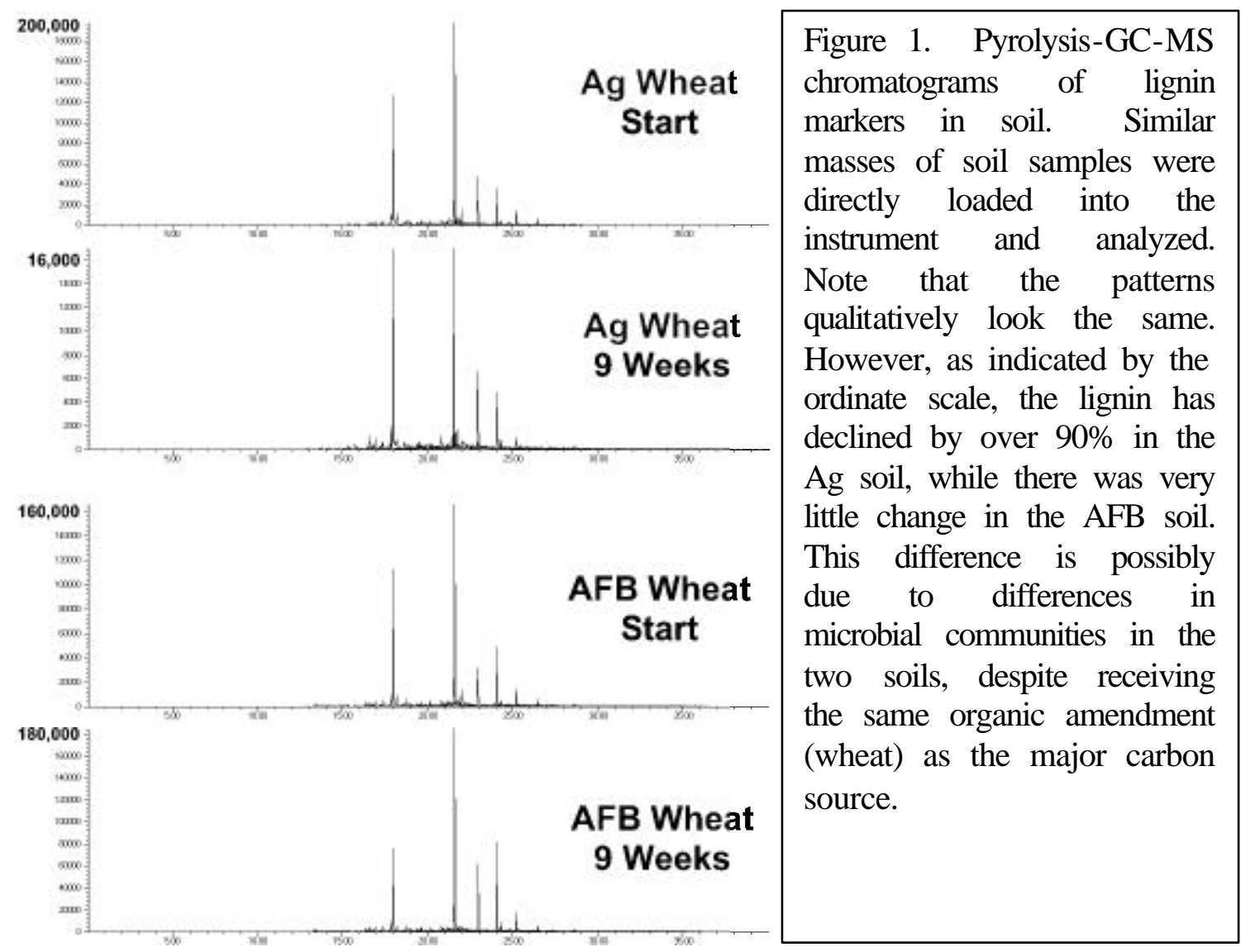

The aged Ag and AFB soils and Cd-loaded wheat roots were provided to the Co-PI (Crowley) for determining the bioavailability of cadmium in plant tissues to rootdecomposing microorganisms from the aged soils. Experiments conducted this first year have focused on examining the structure and diversity of bacterial communities during the degradation of Cd-loaded plant roots, and whether communities that have been preadapted to cadmium stress respond differently to Cd-loaded plant roots as compared to bacteria from low cadmium soils. Secondly, cadmium adapted bacterial communities are thought to have different mechanisms for responding to the presence of $\mathrm{Cd}$ that can in turn affect its long term fate in the environment. These include the production of exopolysaccharides cell capsules to prevent entry of metals into the cells, cadmium efflux systems, and synthesis of cadmium binding proteins. By monitoring the stress adaptations that are present in the community, and the response of the communities to high and low cadmium containing plant materials, we can assess the relative bioavailability of cadimium to the soil bacterial communities.

To examine the bacterial community responses, soils from low cadmium (Ag) and high cadmium (AFB) locations were incubated with high and low cadmium containing plant root tissues by overlaying soil on top of coarsely ground root samples in microcosm experiments. After 4 weeks of incubation, the root tissues were removed from the soil matrix and bacterial community structures were analyzed using PCR-DGGE of $16 \mathrm{~S}$ rDNA. This methodology results in the generation of $16 \mathrm{~S}$ rDNA profiles that can then be analyzed using image analysis and multivariate statistical procedures. Each of the DNA 
bands in the gel represent different groups or species of bacteria that are present in the community. Results of these experiments showed that there were 42 DNA bands representing the predominant root degrading bacterial communities in the $\mathrm{Ag}$ soil, of which 7 were significantly affected by the presence of cadmium in the plant tissues. Six of these 7 bands that responded to the presence of $\mathrm{Cd}$ decreased in intensity, indicating that these bacterial species were adversely affected; whereas 1 band increased in predominance, indicating the growth of a cadmium tolerant strain or group of bacteria that competitively displaced the less tolerant bacterial species. Factor analysis of the community fingerprints from the AFB soil showed an opposite response in which bacterial communities were more diverse on cadmium containing roots than on control root tissues. However, these results are from one time point and provide only an initial insight into the microbial processes that could affect the release of metals during decomposition of plant roots in soil.

\section{Planned Activities}

Detailed structure analysis of HS from organic matter amended soils will be performed using pyrolysis GC-MS, NMR, FT-IR, and 3-D fluorescence. Detailed element analysis of the soil leachates will be conducted by ICP-MS. The HS structure characteristics will be related to the heavy metal leaching profile.

The ${ }^{13} \mathrm{C}$ and ${ }^{15} \mathrm{~N}$-labeled soils will be chased with unlabeled Cd-loaded plant tissues and various organic waste materials so that the effect of organic amendment on humification and mobility of $\mathrm{Cd}$ from plant origin can be determined. The decay rate of ${ }^{13} \mathrm{C}$ and ${ }^{15} \mathrm{~N}$ labels in HS substructures will be followed by pyrolysis GC-MS or phyrolysis isotoperatioing GC-MS along with the leaching rate of $\mathrm{Cd}$ and other metals that are indigenous in the soils.

Experiments are in progress to examine the microbial community dynamics over a 6 wk period during decomposition of high and low cadmium containing root tissues. In this study, changes in the plant tissue content of metallothionein bound cadmium will be assayed simultaneously to determine the recalcitrance of these important organometallic complexes that chelate cadmium and other metal in plant tissues. We will also conduct higher resolution analyses of the microbial communities using DNA microarray methods that will enable us to specifically identify the bacterial genera that are involved in decomposition of heavy metal-containing plant materials. These experiments will also integrate with analyses of organic matter constituents described above, which can provide valuable clues to the functional consequences of microbial activity.

In addition to bacteria, fungi are extremely important in degradation processes and may contribute to stabilization of metals that are bound on to their cell walls or to metallothioneins. Primers for analysis of fungal communities have been developed and will be applied to determine the identity of the fungi that attack Cd-loaded plant materials. The studies being conducted to analyze microbial and fungal processes will continue to be interfaced with analytical determinations of cadmium sorption to biological substances that are produced during decomposition of plant materials. 


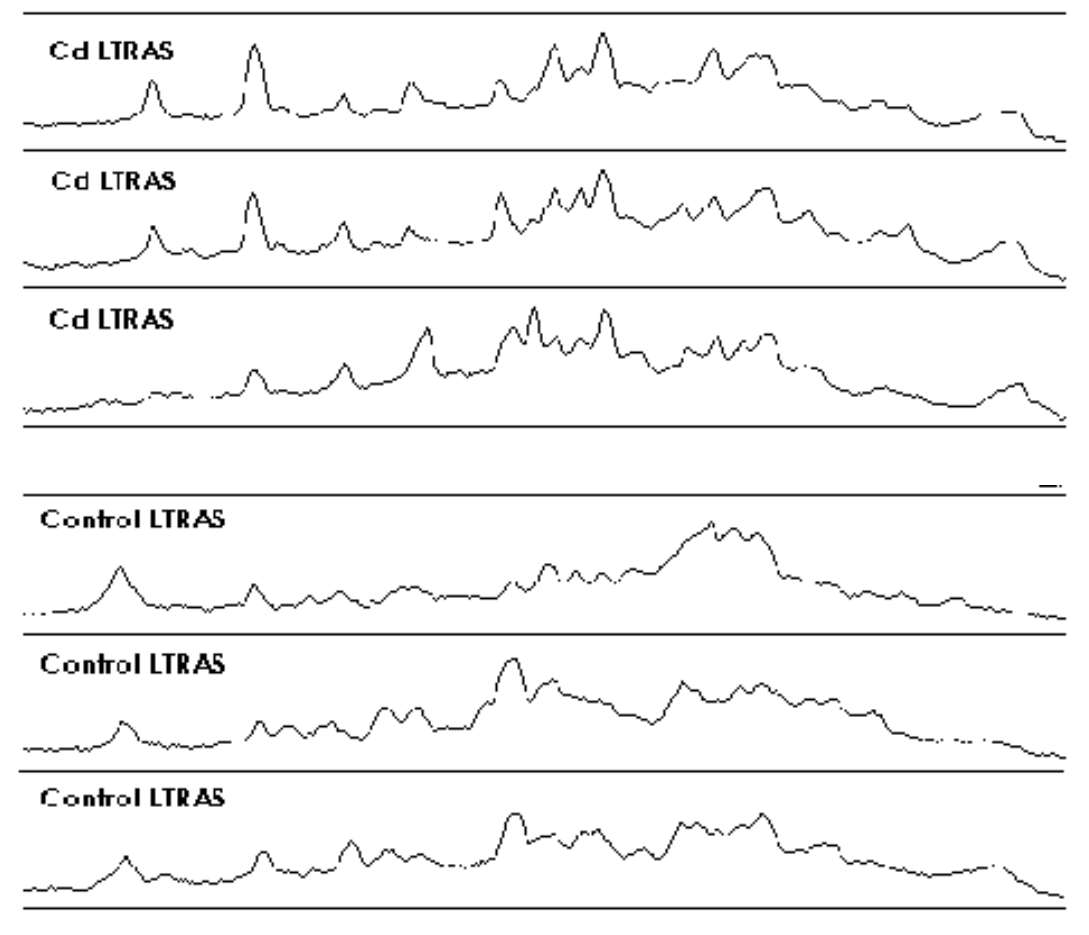

Bacterial community 16S rDNA profiles associated with decomposition of high and low cadmium-containing root tissues after 4 weeks of degradation. Profiles were generated by PCR-DGGE of 16S rDNA, after which statistical differences between communities associated with the treatments were analyzed using peak fitting and multivariate statistical analyses procedures. 